\title{
Autorenverzeichnis 1987
}

Abel, U., 345 Aiginger, P., 302 Ammon, A., 97 Arnold, H., 67

Bacigalupo, A., 172 Bartram, C. R., 127 Battegay, M.. 308 Baumgartner, G., 100 Berger, R., 196 Blossey, H. C, 321 Blöchl-Daum, B., 54 Bornkamm, G. W., 196 Bönisch, E., 104 Brambs, H.-J., 84 Braun, A., 79 Bruggmoser, G., 84 Bruntsch, U., 226 Buchelt, L., 367 Busch, F. W., 157

Büchner, Th., 14

Cafaro, A., 134 Calcanis, A., 376 Congiu, M., 172

Dauer, U., 250 Dennig, D., 57 Diehl, V., 62, 114 Dietrich, M., 290 Doberauer, C, 340 Drings, P., 357, 376

Edler, L., 67, 320 Ehninger, G., 218 Eick, D., 196 Eiermann, W., SI Emmrich, F., 121 Essers, U., 321

Fauser, A. A., 144 Fehr, R., 305 Figari, O., 172 Flechtner, H., 67, 330 Fleischer, K., 367

Fonatsch, Ch., 206 Franke, R. P., 250 Freund, U., 84 Fricke, J., 305 Fritsch, H., 67 Fritz, M., 67

Fritze, D., 67, 345 Fülle, H. H., 57

Gall, F. P., 247 Gallmeier, W. M., 226

Gästner, E., 104 Gelderblom, H., 186 Georgii, A., 62 Gerhartz, H., 62 Geyer, H., 79 Göldel, N., 312 Gradl, G., 206 Graewe, T., 97 Grünert, P., 100

Hain, E., 90 Hans, K., 357 Hartlapp, J., 104 Hartmann, H. R., 232 Hasford, J., 43 Havemann, K., 357 Hehlmann, R., 28 Heim, M. E. 67, 345 Hellriegel, K.-P., 57 Hengastner, H., 232

Herbolsheimer, M., 284 Hermann, M.. 350 Herrmann, R., 67 Heuß, H.,67 Hiddemann, W., 11, 18 Hiemeyer, V., 367 Hiller, E., 62 Ho, A. D., 345 Holle, R., 357 Horaczek, A., 100 HowsonJan, K., 144 Höpken, S., 250

Jakesz, R., 72 Jaspers, L., 312 Jäger, W., 290 Jehn, U., 312

Kabelitz, K., 67 Kalden, J. R., 240 Kanz, B., 144 Kaukel, E., 90 Keminger, K., 350 Kern, P., 50 Kiesewetter, H., 250 Kitz, K., 100

Kleeberg, U. R., 104, 321 Kleihauer, E., 138 Kloiber, R., 367 Kloke, O., 340 Klumpp, D., 367

Knöchelmann, R., 367 Kober, F., 350 Kolb, R., 72 Koschel, G., 90 Köppler, H., 34

Krasemann, E. O., 305 Kremer, G., 294 Krempel, B., 290 Kreuser, E.-D., 367 Kreutzmann, H., 18 Kubista, E., 302 Kurschel, E., 340 Kurth, R., 186

Langer, M., 302 Leichtle, R., 367 Lenoir, G., 196 van Lint., M. T., 172 Lower, J., 186 Löffler, M., 62 Löhr, G. W., 144 Lower, R., 186

Marmont, A., 172 Martinez, C, 324 Maschmeyer, G., 18 Mebes, W., 345 Mende, W., 367

Messerer, D., 43 Meuret, G., 367 Mittermayer, Ch., 250 Müller, C. A., 157

Nagel, G. A. 104. 321 Nawroth, P. P., 254 Neiß, A., 43 Niederle, N., 340 Niethammer, D., 174

Nissen, C, 153

Obrecht, J. P., 308 Obrist, R., 308

Pandolfi,F., 134 Pfreundschuh, M., 62, 144 Piaggio, G., 172 Polack, A., 196 Popp, C, 367

Porzsolt, F., 43, 367 Possinger, K., SI Pötzi, P., 302 Pralle, H., 5

Queißer, U., 324 Queißer, W., 67, 324

Rausch, P., 302 Redenbacher, M, 367 Reiner, A., 72 Reiner, G., 72 Renner. D.. 324 
Richter, E., 284 Ries, G., SI Risau, W., 260 Ritter, J., 24 Romen, W., 284 Rubas, W.,232 Rühl, U., 62

Salzer, H., 302 Sauer, H., 51, 294 Sauerbrei, W., 290 Scarselli, E., 134 Sevelda, P., 302 Smith, K., 62 Spona, J., 302 Supersaxo, A., 232

Scherpe, A., 104 Schmelz, ML, 367 Schmidt, C. G., 340 Schmitt, R., 284 Schnittler, H., 250

Schott, H., 232 Schreml, W., 367 Schroeder, M., 357 Schuler, U., 218 Schünemann, H., SI

Schwendener, R. A., 232

Staffen, A., 302 Stern, D. M. 254 Storb, R., 166 Strigl, P., 367 Stuller, I., 302

Trux, F. A., 67

Vehling-Kaiser, U., 312

Waller, H. D. 157, 218 Wander, H.-E., 104, 321 Wannenmacher, M., 84 Wendt, F., 18 Wildt, L., 290 Willich, N., SI Wilmanns, W., 294 Witherspoon, R., 166 Wolf, M., 357 Wunsch, M., 100 Zacharski, L. R., 264 Zangs, R., 250 Zielinski, C. C, 302 